\title{
Teleportation of atomic states via position measurements
}

\author{
Michele Tumminello ${ }^{1}$ and Francesco Ciccarello ${ }^{1,2,3}$ \\ ${ }^{1}$ Dipartimento di Fisica e Tecnologie Relative, Università di Palermo, Viale delle Scienze, I-90128 Palermo, Itall * $^{*}$ \\ ${ }^{2}$ Consorzio Nazionale Interuniversitario per le Scienze Fisiche della Materia , Italy \\ ${ }^{3}$ NEST and Dipartimento di Scienze Fisiche ed Astronomiche, \\ Università di Palermo, Via Archirafi 36, I-90123 Palermo, Italy
}

\begin{abstract}
We present a scheme for conditionally teleporting an unknown atomic state in cavity QED which requires two atoms and one cavity mode. The translational degrees of freedom of the atoms are taken into account using the optical Stern-Gerlach model. We show that successful teleportation with probability $1 / 2$ can be achieved through local measurements of the cavity photon number and atomic positions. Neither direct projection onto highly entangled states nor holonomous interactiontime constraints are required.
\end{abstract}

PACS numbers: 42.50.-p, 32.80.Lg, 03.65.Ud

\section{INTRODUCTION}

Quantum entanglement, maybe the most intriguing feature of quantum mechanics [1], is a powerful resource for quantum information processing tasks [2].

An outstanding application of entanglement is the teleportation of an unknown qubit, the unit of quantum information, between two systems. In the seminal paper by Bennett et al. [3], a quantum state is transferred from qubit $A$ to qubit $B$ using an ancilla, e.g. a third auxiliary qubit $C$. Qubits $B$ and $C$ are initially prepared in an entangled state. A Bell measurement on $A$ and $C$ is then made. Depending on the outcome of such measurement, a suitable unitary transformation on $B$ is performed in order to reconstruct the initial quantum state of $A$. Teleportation is successful with probability 1 . Soon after the proposal by Bennett et al., quantum teleportation has received considerable attention culminated in its experimental demonstration in a number of works $[4,5,6,6]$.

Cavity QED systems - where Rydberg atoms couple to the quantized electromagnetic (e.m.) field of a superconductive cavity 8] - have received considerable attention during the last years 9]. Cavity QED systems have been proposed for implementing teleportation protocols of internal quantum states between atoms, a task which is particularly attractive especially after its experimental proof for trapped ion systems [10]. Generally speaking, in such cavity QED schemes a quantum internal state is teleported between two atoms via coherent interaction with cavity field modes and/or auxiliary atoms which act as quantum channels.

Quite recently, efforts have been done for achieving teleportation without direct projections onto Bell states [11, 12, 13, 14, 17]. In particular, Zheng has proposed a scheme for approximately teleporting an unknown internal state between two atoms which successively interact with a cavity mode according to the Jaynes-Cummings

*Electronic address: tumminello@lagash.dft.unipa.it
Hamiltonian [13]. The probability of success is $1 / 4$ and only measurements of product states are required. Ye and Guo have presented another scheme that does not require projections onto Bell states and makes use of three atoms and a single-mode cavity field out of resonance [14]. The atom-atom coupling via the virtual excitations of the cavity field is exploited for teleporting a quantum state between two atoms with probability of success $1 / 2$. Ye and Guo presented their work in terms of a "no Bell-state measurement scheme". This parlance was later criticized in a comment by Chhajlany and Wjcik [18] who showed how the scheme by Ye and Guo, despite its use of local measurements, in fact relies on Bell state measurements. Protocols of this sort are indeed more properly classified as methods to achieve teleportation without requiring direct projections onto Bell states [19]. Noticeably, both the schemes by Zheng [13] and Ye and Guo [14] require precise tuning of the atom-cavity field interaction time.

To our knowledge, no cavity QED-teleportation scheme has so far accounted for the translational dynamics of atoms flying through a cavity. Indeed, the spatial structure of the quantum e.m. field along the $x$-cavity axis affects the internal dynamics of a flying atom. This leads to an atom-field coupling constant which in fact depends on the atomic translational degrees of freedom along the $x$-direction. Such circumstance - taking place whenever the atomic wavepacket has a width non negligible with respect to the field wavelength - has been shown to give rise to a number of observable phenomena such as optical Stern-Gerlach effect [20], self-induced transparency 21], modulation of the atomic decay in a damped cavity 22], non-dissipative damping of the Rabi oscillations [23, 24].

It is clear that the involvement of the translational degrees of freedom introduces non-dissipative decoherence in the atom-field dynamics. Such effect, stemming from the entanglement between the atom-field system and the atomic translational degrees of freedom, has been shown to spoil the non-local correlations between two atoms which successively interact with the same cavity mode [25, 26]. Accordingly, the inclusion of the trans- 
lational dynamics is thus expected to decrease the efficiency of those teleportation protocols relying on the coherent atom-cavity mode coupling.

However, a different perspective can be adopted. Indeed, one may wonder whether such additional degrees of freedom could be fruitfully exploited as a resource for attaining efficient atomic teleportation provided that measurements of the atomic positions are performed. According to such a scenario, the atomic translational degrees of freedom play the role of further quantum channels able to transfer information between the internal degrees of freedom of different atoms.

A crucial motivation in the search for such a teleportation protocol is that, according to the optical SternGerlach model, the wavefunction of a two-level atom entering a cavity generally splits into a set of deflected wavepackets, each corresponding to a different atom-field dressed state [23, 27]. For an increasing atom-cavity interaction time, such outgoing wavepackets become more and more distinguishable up to the point that which-path information becomes accessible [24]. This information is used in our protocol for attaining conditional transfer of quantum information between two atoms which successively interact with the same cavity mode. This is indeed the central mechanism underlying the physics presented in this work.

In this paper, we consider two atoms which successively enter the same cavity in either a nodal or antinodal region of the corresponding field mode. Each atom interacts with such mode according to the optical SternGerlach Hamiltonian. This can be approximated as a linear (quadratic) expansion in the atomic position along the cavity axis when a nodal (antinodal) region is considered. Both the atoms are assumed to enter the cavity in a given minimum uncertainty Gaussian wave packet with the target atom and the resonant mode initially in the excited and vacuum state, respectively. We show that conditional teleportation of an internal atomic state can be achieved by local measurements of the atomic positions, the cavity photon-number and the internal state of the atom whose state is to be transmitted. No direct Bell-state measurement is required. We thus prevent the projection of our two-atoms system onto highly entangled subspaces, therefore avoiding the need of (in general quite difficult) joint measurements. This is a major advantage of teleportation schemes that do not rely on direct Bellstate measurements. Furthermore, at variance with other cavity-QED protocols which work without direct Bellstate measurements [13, 14], no holonomous constraints on the atom-cavity interaction times are required. It only suffices that the time of flight of each atom inside the cavity is long enough in order for the outgoing deflected wavepackets to be distinguished with reasonable approximation. We show that successful teleportation of an atomic state can be attained with probability $1 / 2$.

This paper is organized as follows. In Sec. II, we introduce the system and the Hamiltonian both in the nodal and in the antinodal case. In Sec. III, the main part of this work, we describe the teleportation scheme. A relevant property the protocol relies on is the which-path information about the outgoing atomic wave packets. The conditions allowing this information to be accessible are reviewed and discussed in Sec. IV] Finally, in Sec. V] we draw our conclusions.

\section{SYSTEM AND APPROACH}

We consider two identical two-level atoms, labeled 1 and 2, of mass $m$ and Bohr frequency $\omega$. The atoms interact in succession with the e.m. field of the same e.m. cavity. We assume that the velocity of each atom along the $z$-direction (orthogonal to the $x$-cavity axis) is large enough that the motion along the $z$-axis is not affected by the cavity field and can be treated classically. Denoting by $a$ and $a^{\dagger}$ the annihilation and creation operators of the cavity field and assuming the resonance condition, the free Hamiltonian $H_{0}$ can be written as

$$
H_{0}=\sum_{i=1,2}\left[\frac{\hat{p}_{i}^{2}}{2 m}+\hbar \omega S_{z, i}\right]+\hbar \omega a^{\dagger} a
$$

where - for each atom $i=1,2-S_{z, i}, S_{ \pm, i}$ are the usual spin- $1 / 2$ operators and $\hat{p}_{i}=-i \hbar\left(d / d x_{i}\right)$ is the $x$ component of the momentum operator. In the Rotating Wave Approximation, each atom $i$ couples to the cavity field according to the interaction Hamiltonian

$$
H_{i f}=\hbar \varepsilon \sin \left(k \hat{x}_{i}\right)\left(a^{\dagger} S_{-, i}+a S_{+, i}\right) \quad(i=1,2)
$$

with $k$ and $\varepsilon$ standing for the wave number of the e.m. mode and the atom-field coupling constant, respectively, and where $\hat{x}_{i}$ is the $i$ th atomic position operator along the cavity axis.

Hamiltonian (2) accounts for the spatial structure of the e.m. field along the $x$-cavity axis. Rigorously speaking, it should be mentioned that the atom-field coupling constant has also a spatial structure along both the $y$ and $z$-axes perpendicular to the cavity axis. Such structure, having a gaussian profile of the form $\exp \left[-\left(y^{2}+z^{2}\right) / w_{0}^{2}\right]$ ( $w_{0}$ cavity waist) 15], is neglected by the optical SternGerlach interaction Hamiltonian (2). Concerning the $z$ axis, the large atomic velocity along such direction indeed ensures that each flying atom is insensitive to the cavity field and thus to its structure along such axis. On the other hand, we assume to be in the regime such that $w_{0} \gg 2 \pi / k$. In this case, it is enough to take into account only the $x$-structure of the e.m. field, assuming a uniform spatial dependence on the transversal direction. Such a regime is a feasible one given that microwave cavities having a value of $w_{0} \gg 2 \pi / k$ are quite common (see e.g. 16] where $w_{0}$ is as large as $6 \mathrm{~mm}$ ).

When both the atoms enter the cavity in a nodal region of the cavity mode with the width $\sigma_{x_{i}}$ of their respective wavepackets small enough compared to $2 \pi / k$ 
$\left(\sigma_{x_{i}} \ll 2 \pi / k\right), H_{i}$ can be approximated as a linear expansion in the atomic position

$$
H_{i N}=\hbar \varepsilon k \hat{x}_{i}\left(a^{\dagger} S_{-, i}+a S_{+, i}\right),
$$

while in an antinodal region it takes the form

$$
H_{i A}=\hbar \varepsilon\left(1-\frac{k^{2} \hat{x}_{i}^{2}}{2}\right)\left(a^{\dagger} S_{-, i}+a S_{+, i}\right) .
$$

In Eqs. (3) and (4), $\hat{x}_{i}$ stands for the atomic position operator of the $i$ th atom with respect to a nodal point and an antinodal point, respectively.

At time $t=0$, atom 1 enters the cavity and interacts with the field for a time $t_{1}$. At a later time $t_{2}>t_{1}$, atom 2 enters the cavity and couples to the field state modified by the first atom. At time $t_{3}>t_{2}$ atom 2 exits the cavity. At times $t \geq t_{3}$ both the atoms are therefore out of the cavity and evolve freely. In the interaction picture, the Hamiltonian at all times in a nodal region of the cavity field, reads

$$
\begin{aligned}
H_{N}^{I}(t) & =\hbar \varepsilon k\left(\hat{x}_{1}+\frac{\hat{p}_{1}}{m} t\right) \mu_{t}\left(0, t_{1}\right) u_{1} \\
& +\hbar \varepsilon k\left(\hat{x}_{2}+\frac{\hat{p}_{2}}{m} t\right) \mu_{t}\left(t_{2}, t_{3}\right) u_{2}
\end{aligned}
$$

where we have introduced the atom-field operators $u_{i}=$ $a^{\dagger} S_{-, i}+a S_{+, i}$ and where the time interval during which each atom interacts with the cavity mode is accounted for through the function $\mu_{t}\left(t^{\prime}, t^{\prime \prime}\right)=\theta\left(t-t^{\prime}\right)-\theta\left(t-t^{\prime \prime}\right)$, $\theta(t)$ being the usual Heaviside function.

In an antinodal region of the cavity field, the Hamiltonian in the interaction picture takes the form

$$
\begin{aligned}
H_{A}^{I}(t) & =\hbar \varepsilon\left[1-\frac{k^{2}}{2}\left(\hat{x}_{1}+\frac{\hat{p}_{1}}{m} t\right)^{2}\right] \mu_{t}\left(0, t_{1}\right) u_{1} \\
& +\hbar \varepsilon\left[1-\frac{k^{2}}{2}\left(\hat{x}_{2}+\frac{\hat{p}_{2}}{m} t\right)^{2}\right] \mu_{t}\left(t_{2}, t_{3}\right) u_{2} .
\end{aligned}
$$

Of course, in the time interval $\left[t_{1}, t_{2}\right]$ and for $t \geq t_{3}$ both $H_{N}^{I}(t)$ and $H_{A}^{I}(t)$ vanish since no atom is inside the cavity. The Hamiltonian operators of Eqs. (5) and (6) can be used to derive the exact dynamics of a given initial state of the two-atom-field system at times $t \geq t_{3}$. This is accomplished through the respective evolution operators $U_{\alpha}^{I}\left(t \geq t_{3}\right)$

$$
U_{\alpha}^{I}\left(t \geq t_{3}\right)=T \exp \left[-\frac{i}{\hbar} \int_{0}^{t_{3}} H_{\alpha}^{I}(t) d t\right] \quad(\alpha=N, A)
$$

with $T$ standing for the time-ordering operator and where the second integration bound is due to the fact that $H_{\alpha}^{I}=$ 0 for $t \geq t_{3}$.

Due to the fact that atom 2 enters the cavity after atom 1 has come out of it, it is possible to split up $U_{\alpha}^{I}\left(t \geq t_{3}\right)$ into the product of two evolution operators $U_{\alpha, 1}^{I}\left(t \geq t_{3}\right)$ and $U_{\alpha, 2}^{I}\left(t \geq t_{3}\right)(\alpha=N, A)$. Each operator $U_{\alpha, i}^{I}\left(t \geq t_{3}\right)$ only affects the dynamics of atom $i$. In formulae (from now on, whenever unnecessary, the time argument " $t \geq$ $\left.t_{3}\right)$ " and/or the apex " $I$ " in the evolution operators will be omitted)

$$
U_{\alpha}=U_{\alpha, 2} \cdot U_{\alpha, 1} \quad(\alpha=N, A)
$$

with

$$
\begin{aligned}
& U_{\alpha, 1}=T \exp \left[-\frac{i}{\hbar} \int_{0}^{t_{1}} H_{\alpha}^{I}(t) d t\right]=U_{\alpha, 1}\left(\hat{x}_{1}, \hat{p}_{1}, u_{1}\right) \\
& U_{\alpha, 2}=T \exp \left[-\frac{i}{\hbar} \int_{t_{2}}^{t_{3}} H_{\alpha}^{I}(t) d t\right]=U_{\alpha, 2}\left(\hat{x}_{2}, \hat{p}_{2}, u_{2}\right),
\end{aligned}
$$

where in the right-hand side of both equations we have explicitly indicated the operators each $U_{\alpha, i}$ depends on according to Eqs. (5) and (6).

\section{TELEPORTATION SCHEME}

We denote the ground and excited states of the $i$ th atom by $\left|g_{i}\right\rangle$ and $\left|e_{i}\right\rangle$, respectively. Assume that atom 2 is the one whose initial internal state, say $\left|\alpha_{2}\right\rangle$, is to be teleported. Such state is written as

$$
\left|\alpha_{2}\right\rangle=\cos \frac{\vartheta}{2}\left|e_{2}\right\rangle+e^{i \varphi} \sin \frac{\vartheta}{2}\left|g_{2}\right\rangle
$$

with $\vartheta \in[0, \pi]$ and $\varphi \in[0, \pi]$.

By indicating the Fock states of the cavity field as $|n\rangle$ $(n=0,1, \ldots)$, we consider the following initial state of the system:

$$
|\Psi(0)\rangle=\left|\varphi_{1}(0)\right\rangle\left|e_{1}\right\rangle\left|\varphi_{2}(0)\right\rangle\left|\alpha_{2}\right\rangle|0\rangle,
$$

where $\left|\varphi_{i}(0)\right\rangle$ (associated with each atom $i=1,2$ ) is a Gaussian wavepacket of minimum uncertainty, such that the product between the initial position and momentum widths fulfills $\sigma_{x_{i}} \cdot \sigma_{p_{i}}=\hbar / 2$.

Consider now the usual dressed states of the $i$ th atom $\left|\chi_{n, i}^{ \pm}\right\rangle=\left(\left|e_{i}\right\rangle|n\rangle \pm\left|g_{i}\right\rangle|n+1\rangle\right) / \sqrt{2}(n=0,1, \ldots)$. These states are eigenstates of the $u_{i}$ operators since $u_{i}\left|\chi_{n, i}^{ \pm}\right\rangle=$ $\pm \sqrt{n+1}\left|\chi_{n, i}^{ \pm}\right\rangle$(while $\left.u_{i}\left|g_{i}\right\rangle|0\rangle=0\right)$. The dressed states together with $\left|g_{i}\right\rangle|0\rangle(i=1,2)$ represent an orthonormal basis of the corresponding Hilbert space. It is important to notice that $u_{i}$ commutes with $U_{\alpha, i}$ according to Eqs. 9 and 10) and the corresponding Hamiltonian operators of Eqs. (5)and 6). It follows that the effective representation $U_{\alpha, i}^{(n, \pm)}$ of $U_{\alpha, i}$, as applied to a dressed state $\left|\chi_{n, i}^{ \pm}\right\rangle$, is obtained by simply replacing $u_{i}$ with $\pm \sqrt{n+1}$ in Eqs. (9) and (10). This yields

$$
U_{\alpha, i}^{(n, \pm)}=U_{\alpha, i}\left(\hat{x}_{i}, \hat{p}_{i}, \pm \sqrt{n+1}\right) \quad(n=0,1, \ldots),
$$

while the effective representation of $U_{N, i}-$ as applied to state $\left|g_{i}\right\rangle|0\rangle$ - reduces to the identity operator for both the atoms $i=1,2$. 
The operators in Eq. (13) clearly affect only the atomic translational dynamics and therefore allow to define a family of atomic translational wavepackets $\left|\Phi_{\alpha, n, i}^{ \pm}\right\rangle$according to

$$
\left|\Phi_{\alpha, n, i}^{ \pm}\right\rangle=U_{\alpha, i}^{(n, \pm)}\left|\varphi_{i}(0)\right\rangle
$$

such that

$$
U_{\alpha, i}\left|\varphi_{i}(0)\right\rangle\left|\chi_{n, i}^{ \pm}\right\rangle=\left|\Phi_{\alpha, n, i}^{ \pm}\right\rangle\left|\chi_{n, i}^{ \pm}\right\rangle .
$$

Once the time evolution operator (8) is applied to $|\Psi(0)\rangle$, the state of the whole system at a time $t \geq t_{3}$ - when both the atoms are out of the cavity - can be written in the form (from now on, the index $\alpha$ in the $\Phi$ states will be omitted)

$$
\begin{aligned}
\left|\psi\left(t_{3}\right)\right\rangle= & \left|\lambda_{0,1}\right\rangle\left|\varphi_{2}(0)\right\rangle\left|g_{2}\right\rangle|0\rangle \\
& +\sum_{n=0,1} \sum_{\eta=-,+}\left(\left|\lambda_{n, 1}^{\eta}\right\rangle\left|\Phi_{n, 2}^{\eta}\right\rangle\left|\chi_{n, 2}^{\eta}\right\rangle\right),
\end{aligned}
$$

where the $\lambda$ states of atom 1 are defined according to

$$
\begin{aligned}
\left|\lambda_{0,1}\right\rangle= & \left(\frac{\left|\Phi_{0,1}^{+}\right\rangle+\left|\Phi_{0,1}^{-}\right\rangle}{2}\right) e^{i \varphi} \sin \frac{\vartheta}{2}\left|e_{1}\right\rangle, \\
\left|\lambda_{0,1}^{ \pm}\right\rangle= & \left(\frac{\left|\Phi_{0,1}^{+}\right\rangle+\left|\Phi_{0,1}^{-}\right\rangle}{2 \sqrt{2}}\right) \cos \frac{\vartheta}{2}\left|e_{1}\right\rangle \\
& \pm\left(\frac{\left|\Phi_{0,1}^{+}\right\rangle-\left|\Phi_{0,1}^{-}\right\rangle}{2 \sqrt{2}}\right) e^{i \varphi} \sin \frac{\vartheta}{2}\left|g_{1}\right\rangle, \\
\left|\lambda_{1,1}^{ \pm}\right\rangle= & \left(\frac{\left|\Phi_{0,1}^{+}\right\rangle-\left|\Phi_{0,1}^{-}\right\rangle}{2 \sqrt{2}}\right) \cos \frac{\vartheta}{2}\left|g_{1}\right\rangle .
\end{aligned}
$$

The procedure for obtaining state $\left|\psi\left(t_{3}\right)\right\rangle$ is detailed in Appendix A. In what follows, we shall indicate the time spent inside the cavity by atoms 1 and 2 with $\tau_{1}=t_{2}-t_{1}$ and $\tau_{2}=t_{3}-t_{2}$ respectively. The states $\left|\Phi_{n, i}^{ \pm}\right\rangle$appearing in Eq. (16) fulfill the following important property both in the nodal and antinodal case [24, 25, 26]

$$
\lim _{\tau_{i} \rightarrow \infty}\left\langle\Phi_{n, i}^{+} \mid \Phi_{n, i}^{-}\right\rangle=0 .
$$

Such property, together with the features of the outgoing wavepackets $\left|\Phi_{n, i}^{+}\right\rangle$, is discussed in Sec. IV.

According to Eq. (20), wavepackets $\left|\Phi_{n, i}^{+}\right\rangle$and $\left|\Phi_{n, i}^{-}\right\rangle$ exhibit a negligible overlap for long enough times of flight $\tau_{i}$. As shown in Refs. 25, 26], times of flight of the order of a few Rabi oscillations are sufficient in order to get negligible overlapping [28].

Such outstanding circumstance makes it possible to distinguish the elements of the set of translational states $\left\{\left|\Phi_{n, i}^{ \pm}\right\rangle\right\}$through measurements of the atomic positions along the $x$-axis [29].

It is straightforward to show that Eq. (20) implies that all the terms appearing in (16) are orthogonal provided that $\tau_{1}$ and $\tau_{2}$ are sufficiently large.

Once the dressed states $\left|\chi_{n, 2}^{ \pm}\right\rangle$appearing in Eq. (16) are rewritten in terms of states $\left|g_{2}\right\rangle|n\rangle$ and $\left|e_{2}\right\rangle|n\rangle$, one recognizes the occurrence of cases where measurements of the photon number, of the internal state of atom 2 and of the positions of the two atoms can make atom 1 collapse into the initial internal state of atom 2 [Eq. (11)]. Namely a successful teleportation can take place. For instance, the projection of $\left|\psi\left(t_{3}\right)\right\rangle$ onto the the cavity field state $|1\rangle$ gives

$$
\begin{aligned}
\left\langle 1 \mid \psi\left(t_{3}\right)\right\rangle= & {\left[\frac{\left(\left|\Phi_{0,1}^{+}\right\rangle+\left|\Phi_{0,1}^{-}\right\rangle\right)\left(\left|\Phi_{0,2}^{+}\right\rangle-\left|\Phi_{0,2}^{-}\right\rangle\right)}{4} \cos \frac{\vartheta}{2}\left|e_{1}\right\rangle+\frac{\left(\left|\Phi_{0,1}^{+}\right\rangle-\left|\Phi_{0,1}^{-}\right\rangle\right)\left(\left|\Phi_{0,2}^{+}\right\rangle+\left|\Phi_{0,2}^{-}\right\rangle\right)}{4} e^{i \varphi} \sin \frac{\vartheta}{2}\left|g_{1}\right\rangle\right]\left|g_{2}\right\rangle } \\
& +\left[\frac{\left(\left|\Phi_{0,1}^{+}\right\rangle-\left|\Phi_{0,1}^{-}\right\rangle\right)\left(\left|\Phi_{1,2}^{+}\right\rangle+\left|\Phi_{1,2}^{-}\right\rangle\right)}{4} \cos \frac{\vartheta}{2}\left|g_{1}\right\rangle\right]\left|e_{2}\right\rangle .
\end{aligned}
$$

This outcome occurs with probability $(3+\cos \vartheta) / 8$. Assume now that a further measurement of the internal state of atom 2 is made. If the outcome of such measurement is $\left|e_{2}\right\rangle$, atom 1 is projected onto the ground state $\left|g_{1}\right\rangle$ and thus no teleportation of the initial state of atom 2 has occurred. The unconditional probability for this event is calculated to be $(1+\cos \vartheta) / 8$.

However, it can be noticed that if atom 2 is found in the ground state $\left|g_{2}\right\rangle$ a further measurement of the atomic positions with outcomes $\left|\Phi_{0,1}^{+}\right\rangle\left|\Phi_{0,2}^{+}\right\rangle$or $\left|\Phi_{0,1}^{-}\right\rangle\left|\Phi_{0,2}^{-}\right\rangle$ projects atom 1 into the state $\left|\alpha_{1}\right\rangle=\cos \frac{\vartheta}{2}\left|e_{1}\right\rangle+$ $e^{i \varphi} \sin \frac{\vartheta}{2}\left|g_{1}\right\rangle$. This means that state $\left|\alpha_{2}\right\rangle$ of Eq. (11) has been in fact teleported into atom 1.

On the other hand, when the wavepackets $\left|\Phi_{0,1}^{+}\right\rangle\left|\Phi_{0,2}^{-}\right\rangle$ or $\left|\Phi_{0,1}^{-}\right\rangle\left|\Phi_{0,2}^{+}\right\rangle$are found (after that the state $\left|g_{2}\right\rangle$ has been measured) atom 1 collapses into the state

$$
\left|\alpha_{1}^{\prime}\right\rangle=\cos \frac{\vartheta}{2}\left|e_{1}\right\rangle-e^{i \varphi} \sin \frac{\vartheta}{2}\left|g_{1}\right\rangle,
$$

which can be easily transformed into (11) through a 180 degree rotation around the $z$-axis in order to faithfully reproduce the initial state of atom 2 and complete the teleportation. Of course, rigorously speaking, the measurements of the atomic positions do not formally corre- 


\begin{tabular}{||c|c|c|c|c|c|c||}
\hline Photons & Atom 2 & Path atom 1 & Path atom 2 & Teleportation & Internal state atom 1 & Failure probability \\
\hline 2 & - & - & - & Unsuccessful & - & $\frac{1}{8}(1+\cos \vartheta)$ \\
\hline \multirow{6}{*}{1} & $\left|e_{2}\right\rangle$ & - & - & Unsuccessful & - & $\frac{1}{8}(1+\cos \vartheta)$ \\
\cline { 2 - 8 } & $\left|g_{2}\right\rangle$ & $l_{1}^{-}$ & $l_{2}^{-}$ & Successful & $\cos \frac{\vartheta}{2}\left|e_{1}\right\rangle+e^{i \varphi} \sin \frac{\vartheta}{2}\left|g_{1}\right\rangle$ & - \\
\cline { 2 - 8 } & $\left|g_{2}\right\rangle$ & $l_{1}^{-}$ & $l_{2}^{+}$ & Successful $[30]$ & $\cos \frac{\vartheta}{2}\left|e_{1}\right\rangle-e^{i \varphi} \sin \frac{\vartheta}{2}\left|g_{1}\right\rangle$ & - \\
\cline { 2 - 8 } & $\left|g_{2}\right\rangle$ & $l_{1}^{+}$ & $l_{2}^{+}$ & Successful & $\cos \frac{\vartheta}{2}\left|e_{1}\right\rangle+e^{i \varphi} \sin \frac{\vartheta}{2}\left|g_{1}\right\rangle$ & - \\
\cline { 2 - 8 } & $\left|g_{2}\right\rangle$ & $l_{1}^{+}$ & $l_{2}^{-}$ & Successful $[30]$ & $\cos \frac{\vartheta}{2}\left|e_{1}\right\rangle-e^{i \varphi} \sin \frac{\vartheta}{2}\left|g_{1}\right\rangle$ & - \\
\hline \multirow{5}{*}{0} & $\left|g_{2}\right\rangle$ & - & - & Unsuccessful & & $-\frac{1}{4}(1-\cos \vartheta)$ \\
\cline { 2 - 8 } & $\left|e_{2}\right\rangle$ & $l_{1}^{-}$ & $l_{2}^{-}$ & Successful & $\cos \frac{\vartheta}{2}\left|e_{1}\right\rangle+e^{i \varphi} \sin \frac{\vartheta}{2}\left|g_{1}\right\rangle$ & - \\
\cline { 2 - 7 } & $\left|e_{2}\right\rangle$ & $l_{1}^{-}$ & $l_{2}^{+}$ & Successful $[30]$ & $\cos \frac{\vartheta}{2}\left|e_{1}\right\rangle-e^{i \varphi} \sin \frac{\vartheta}{2}\left|g_{1}\right\rangle$ & - \\
\cline { 2 - 7 } & $\left|e_{2}\right\rangle$ & $l_{1}^{+}$ & $l_{2}^{+}$ & Successful & $\cos \frac{\vartheta}{2}\left|e_{1}\right\rangle+e^{i \varphi} \sin \frac{\vartheta}{2}\left|g_{1}\right\rangle$ & - \\
\cline { 2 - 7 } & $\left|e_{2}\right\rangle$ & $l_{1}^{+}$ & $l_{2}^{-}$ & Successful $[30]$ & $\cos \frac{\vartheta}{2}\left|e_{1}\right\rangle-e^{i \varphi} \sin \frac{\vartheta}{2}\left|g_{1}\right\rangle$ & - \\
\hline \multirow{6}{*}{} & & & & & - \\
\hline
\end{tabular}

TABLE I: Teleportation measurement scheme. Each case is represented by given outcomes of the number of photons $\left(1^{s t}\right.$ column), the internal state of atom 2 ( $2^{\text {nd }}$ column), and the paths along which the two atoms are found ( $3^{\text {th }}$ and $4^{\text {th }}$ columns). In the $5^{\text {th }}$ column it is indicated whether or not teleportation has been successful. If successful, the state onto which atom 1 is projected $\left(\left|\alpha_{1}\right\rangle\right.$ or $\left.\left|\alpha_{1}^{\prime}\right\rangle\right)$ is presented ( $6^{\text {th }}$ column). If unsuccessful, the associated unconditional failure probability is given in the last column.

spond to projections onto states $\left|\Phi_{0, i}^{+}\right\rangle$and $\left|\Phi_{0, i}^{-}\right\rangle$. However, due to the discussed orthogonality of $\left|\Phi_{0, i}^{+}\right\rangle$and $\left|\Phi_{0, i}^{-}\right\rangle$, such translational states can be associated with different atomic paths $l_{i}^{+}$and $l_{i}^{-}$. The measurements of the atomic positions cause indeed effective projections on such paths.

Note that the above teleportation scheme, conditioned to the outcome $\left|g_{2}\right\rangle|1\rangle$, is invariant for a change of each $l_{i}^{+}$into $l_{i}^{-}$and vice-versa. This implies that for each atom $i=1,2$ the labeling of the two paths is arbitrary. If both the atoms are found in a path "+" or in a path "-", atom 1 is projected into state (11). If the paths of the two atoms have different signs, regardless of which atom is in which path, state (22) is obtained and the teleportation process can be finalized once a 180 degree rotation on the internal state of atom 1 is applied.

In a similar way, it turns out that, when the field vacuum state $|0\rangle$ is found, the outcome $\left|g_{2}\right\rangle$ cannot transfer the initial state of atom 2 into atom 1 , while successful teleportation is attained when atom 2 is found to be in

The total failure probability, obtained as the sum of the unconditioned failure probabilities (last column of Table I), is $1 / 2$. Teleportation is thus successful with probability $1 / 2$.

Remarkably, notice that only local measurements on the two atoms and the cavity field are required in order to complete the teleportation. Direct projections onto highly entangled states are therefore avoided in our the excited state $\left|e_{2}\right\rangle$. As in the case $\left|g_{2}\right\rangle|1\rangle$, when the atoms are found in the same quantum path (i.e. $l_{1}^{+}$and $l_{2}^{+}$or $l_{1}^{-}$and $l_{2}^{-}$) the first atom is projected into $\left|\alpha_{1}\right\rangle$. Again, when different quantum paths are found (i.e. $l_{1}^{+}$ and $l_{2}^{-}$or $l_{1}^{-}$and $l_{2}^{+}$) teleportation can be finalized after a 180 degree rotation around the $z$-axis. Due to conservation of $\sum_{i=1,2} S_{z, i}+a^{\dagger} a$, no teleportation is possible when the field is found to be in $|2\rangle$.

All the possible outcomes of the protocol are summarized in Table I. For each case - corresponding to given outcomes of the number of photons $\left(1^{\text {st }}\right.$ column), the internal state of atom 2 ( $2^{\text {nd }}$ column), and the paths along which the two atoms are found ( $3^{\text {th }}$ and $4^{\text {th }}$ columns) - it is shown whether or not teleportation has been successful ( $5^{\text {th }}$ column). If successful, the state onto which atom 1 is projected $\left(\left|\alpha_{1}\right\rangle\right.$ or $\left.\left|\alpha_{1}^{\prime}\right\rangle\right)$ is presented (6 $6^{\text {th }}$ column). If unsuccessful, the associated unconditional failure probability is given (last column). A schematic diagram of the teleportation protocol is presented in Fig. 1].

scheme. In Appendix B, we develop a more detailed analysis of the mechanism behind the scheme.

Finally, unlike previous cavity QED protocols not requiring direct Bell-state measurements [13, 14], the interaction time of each atom with the cavity does not need to fulfill any holonomous constraint. It is only required that it is large enough in order for (20) to hold with reasonable approximation. It should be noted that a problem 


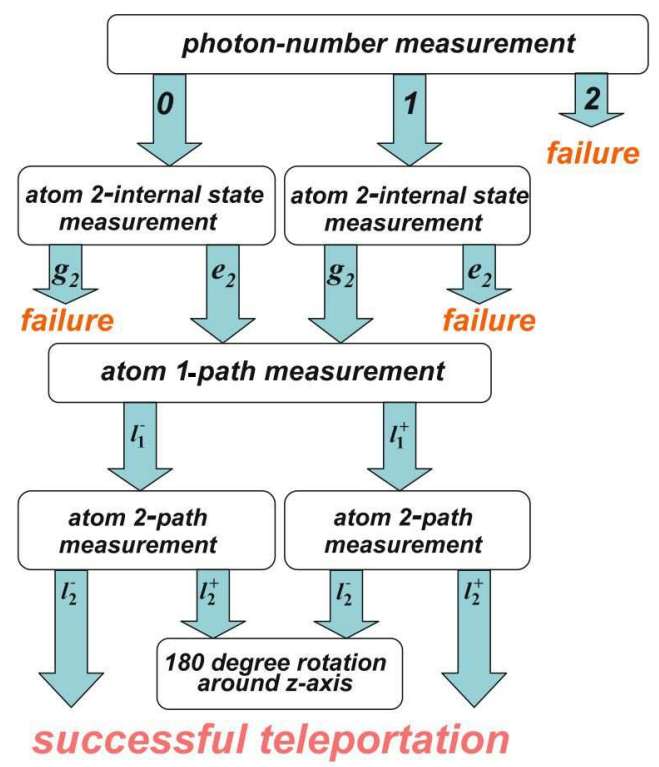

FIG. 1: (Color online) Schematic diagram of the teleportation protocol.

might arise for the implementation of the present scheme given that cavity-photon-number measurements typically require fine tuning of the interaction times between the field and probe atoms. In Appendix C, we show how the atomic which-path information can be exploited in order to accomplish photon-number measurements that require non holonomous constraints.

\section{ORTHOGONALITY OF THE OUTGOING ATOMIC WAVEPACKETS AND WHICH-PATH INFORMATION}

In this section, we discuss in more details the features of the translational states introduced in Eq. (14) and the conditions for which path information to be accessible.

In the nodal case, using Eqs. (5), (9), (10) and (14), the outgoing translational wavepackets $\left|\Phi_{n, i}^{ \pm}\right\rangle$take the form

$$
\begin{aligned}
\left|\Phi_{n, 1}^{ \pm}\right\rangle= & U_{N, 1}^{(n, \pm)}\left|\varphi_{1}(0)\right\rangle= \\
= & \exp \left[i \hbar \frac{\varepsilon^{2} k^{2}}{12 m}(n+1) t_{1}^{3}\right] \\
& \cdot \exp \left[\mp i \varepsilon k \sqrt{n+1} t_{1}\left(\hat{x}_{1}+\frac{\hat{p}_{1}}{2 m} t_{1}\right)\right]\left|\varphi_{1}(0)\right\rangle,
\end{aligned}
$$

and

$$
\begin{aligned}
\left|\Phi_{n, 2}^{ \pm}\right\rangle= & U_{N, 2}^{(n, \pm)}\left|\varphi_{2}(0)\right\rangle= \\
= & \exp \left\{\mp i \varepsilon k \sqrt{n+1}\left(t_{3}-t_{2}\right)\left[\hat{x}_{2}+\frac{\hat{p}_{2}}{2 m}\left(t_{3}+t_{2}\right)\right]\right\} \\
& \cdot \exp \left[i \hbar \frac{\varepsilon^{2} k^{2}}{12 m}(n+1)\left(t_{3}-t_{2}\right)^{3}\right]\left|\varphi_{2}(0)\right\rangle .
\end{aligned}
$$

Using Eqs. (23) and (24), it can be shown that [23, 24, 25]

$$
\begin{gathered}
\left\langle\Phi_{n, i}^{+} \mid \Phi_{n, i}^{-}\right\rangle\left(\tau_{i}\right)=\exp \left[-i\left(2 \varepsilon k \sqrt{n+1} x_{0, i}\right) \tau_{i}\right] . \\
\cdot \exp \left[-(n+1)\left(\frac{\hbar \varepsilon k}{m}\right)\left(\frac{\tau_{i}^{2}}{8 \sigma_{x_{i}}^{2}}+\frac{4 m^{2}}{8 \sigma_{p_{i}}^{2}}\right) \tau_{i}^{2}\right],
\end{gathered}
$$

where $x_{0, i}$ stands for the initial average value of the atomic position along the cavity axis. Eq. (25) clearly shows the presence of a damping factor which causes the scalar products $\left\langle\Phi_{n, i}^{+} \mid \Phi_{n, i}^{-}\right\rangle$to vanish at long times. This proves Eq. (20) in the nodal case.

Such behavior, which is at the origin of the nondissipative damping of the Rabi oscillations [23, 24], arises from the increasing distance in the phase space [31] of the deflected outgoing components $\left|\Phi_{n, i}^{ \pm}\right\rangle$of the incoming wavepacket $\left|\varphi_{i}(0)\right\rangle$ [32]. To better highlight this phenomenon, Eq. (25) can indeed be rewritten in the form [23] (from now on, the subscript $i$ will be omitted for simplicity)

$$
\begin{aligned}
\left\langle\Phi_{n}^{+} \mid \Phi_{n}^{-}\right\rangle(\tau)= & \exp \left[-i \Omega_{n}(\tau) \tau\right] \exp \left\{-\frac{\left[x_{n}^{+}(\tau)-x_{n}^{-}(\tau)\right]^{2}}{8 \sigma_{x}^{2}}\right. \\
& \left.-\frac{\left[p_{n}^{+}(\tau)-p_{n}^{-}(\tau)\right]^{2}}{8 \sigma_{p}^{2}}\right\}
\end{aligned}
$$

with

$$
\begin{aligned}
& \Omega_{n}(\tau)=2 k \varepsilon \sqrt{n+1}\left(x_{0}+\frac{p_{0}}{2 m} \tau\right), \\
& x_{n}^{ \pm}(\tau)=x_{0}+\frac{p_{0}}{m} \tau \mp \frac{\hbar k \epsilon}{2 m} \sqrt{n+1} \tau^{2}, \\
& p_{n}^{ \pm}(\tau)=p_{0} \mp \hbar k \varepsilon \sqrt{n+1} \tau .
\end{aligned}
$$

Here $p_{0}$ stands for the initial average momentum. The above equations show that wavepackets $\left|\Phi_{n}^{+}\right\rangle$and $\left|\Phi_{n}^{-}\right\rangle$ respectively represent negatively and positively deflected components of the input wavepacket, the deflection getting larger as $n$ and/or the atom-cavity interaction time $\tau$ grow. This is the reason why, when the interaction time of each atom with the cavity is large enough, whichpath information becomes accessible so that the quantum paths associated with states $\left|\Phi_{n}^{ \pm}\right\rangle$can be distinguished (see Sec. III). In order to better illustrate such effect, we consider an atom of mass $m=10^{-26} \mathrm{~kg}$ entering a microwave cavity in a nodal region. Assume that the initial translational state of the atom is a Gaussian wavepacket of width $\sigma_{x}=\lambda / 10\left(\lambda=2 \pi / k=10^{-5} \mathrm{~m}\right)$ with $x_{0}=p_{0}=0$ and that the atom-field coupling constant $\varepsilon=10^{5} \mathrm{sec}^{-1}$. The resulting quantum paths $l^{ \pm}$ associated with wavepackets $\left|\Phi_{0}^{ \pm}\right\rangle$(i.e. those involved in the teleportation scheme) are shown in Fig. 2 together with their widths $\sigma_{l^{ \pm}}$(i.e. the standard deviations of $\left|\left\langle x \mid \Phi_{0}^{ \pm}\right\rangle\right|^{2}$ ) as functions of the rescaled atom-cavity interaction time $\varepsilon \tau$. Notice that the deflection of the two outgoing paths increase as $\varepsilon \tau$ is raised up to the point that for atom-cavity interaction times larger than $\simeq 6 / \varepsilon$ the two paths can be reliably distinguished through position measurements. Even fewer Rabi oscillations are 


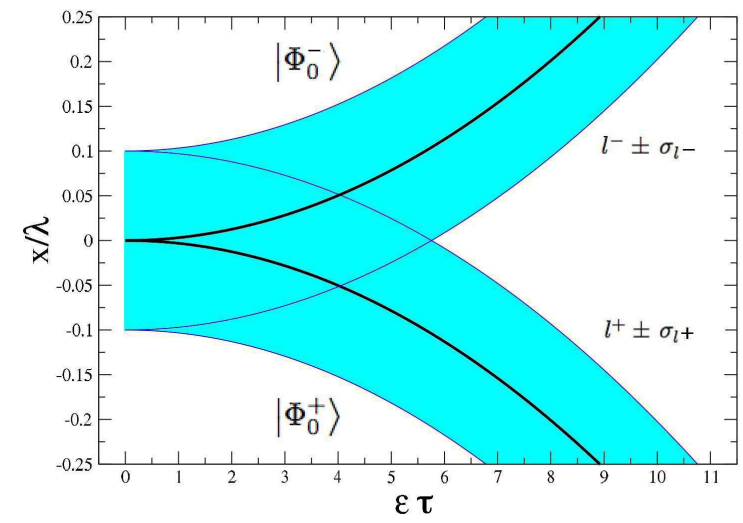

FIG. 2: (Color online) Quantum paths $l^{+} \pm \sigma_{l^{+}}$and $l^{-} \pm$ $\sigma_{l^{-}}$, associated with wavepackets $\left|\Phi_{0}^{ \pm}\right\rangle$, versus the rescaled atom-cavity interaction time $\varepsilon \tau$. The parameters used are: $\lambda=10^{-5} \mathrm{~m}, \varepsilon=10^{5} \mathrm{sec}^{-1}, m=10^{-26} \mathrm{~kg}, \sigma_{x}=\lambda / 10$ and $x_{0}=p_{0}=0$.

needed in order for the orthogonality of $\left|\Phi_{0}^{+}\right\rangle$and $\left|\Phi_{0}^{-}\right\rangle$ to be achieved. This is shown in Fig. 3 where the distinguishability $D$, according to the Englert's definition [33], is plotted as a function of $\varepsilon \tau$. In the present case, $D$ take the form [24]

$$
D=\sqrt{\left(1-\left|\left\langle\Phi_{0}^{+} \mid \Phi_{0}^{-}\right\rangle\right|^{2}\right)} .
$$

Notice that $D>95 \%$ already for $\varepsilon \tau>1.2$. The

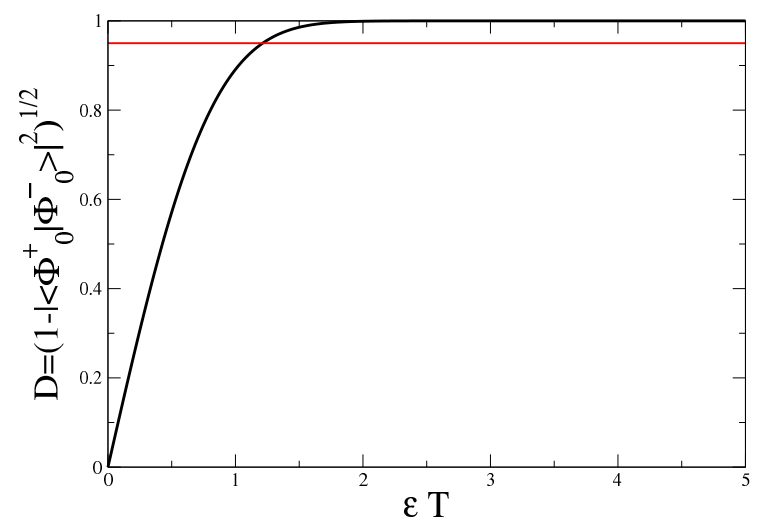

FIG. 3: (Color online) Distinguishability $D$ as a function of the rescaled atom-cavity interaction time $\varepsilon \tau$. When $D=$ 1 the which-path information is completely accessible. The parameters used are: $\lambda=10^{-5} \mathrm{~m}, \varepsilon=10^{5} \mathrm{sec}^{-1}, m=10^{-26}$ $\mathrm{kg}, \sigma_{x}=\lambda / 10$ and $x_{0}=p_{0}=0$.

scalar product $\left\langle\Phi_{0}^{+} \mid \Phi_{0}^{-}\right\rangle$therefore takes less time to vanish $(\simeq 1.2 / \varepsilon)$ than that required for distinguishing the atomic position associated with each path $(\simeq 6 / \varepsilon)$. The reason of such behaviour is that, according to Eq. (26), the damping of $\left\langle\Phi_{0}^{+} \mid \Phi_{0}^{-}\right\rangle$is due to the trajectories in both the position and momentum space. This suggests that momentum, rather than position, measurements might be more suitable in order to acquire the which-path information for some values of the parameters. Property (20) holds in the antinodal case as well. Indeed, using Eqs. (6), (9), (10) and (14), it turns out that, analogously to the nodal case, each scalar product $\left\langle\Phi_{n}^{+} \mid \Phi_{n}^{-}\right\rangle(\tau)$ is always proportional to a damping factor. For instance, in the case $n=1$ it can be calculated as 26]

$$
\begin{array}{r}
\left\langle\Phi_{1}^{+}(\tau) \mid \Phi_{1}^{-}(\tau)\right\rangle=e^{i \frac{\omega_{0}}{2} \tau} e^{-i\left(a_{1}^{2}+b_{1}^{2}\right) \frac{\sin \left(\omega_{0} \tau\right)}{\cosh \left(\omega_{0} \tau\right)}} . \\
\cdot e^{\frac{i}{2} \tanh \left(\omega_{0} \tau\right)\left[\left(a_{1}^{2}-b_{1}^{2}\right)\left(1+\cos \left(2 \omega_{0} \tau\right)\right)+2 a_{1} b_{1} \sin \left(2 \omega_{0} \tau\right)\right]} . \\
\cdot \frac{1}{\sqrt{\cosh \left(\omega_{0} \tau\right)}} e^{-\left(a_{1}^{2}+b_{1}^{2}\right)\left(1-\frac{\cos \left(\omega_{0} \tau\right)}{\cosh \left(\omega_{0} \tau\right)}\right)} . \\
\propto e^{-\tanh \left(\omega_{0} \tau\right)\left[a_{1} b_{1}\left(1-\cos \left(2 \omega_{0} \tau\right)\right)+\frac{1}{2}\left(a_{1}^{2}-b_{1}^{2}\right) \sin \left(2 \omega_{0} \tau\right)\right]} \\
\propto\left[1-\frac{\left(\omega_{0} \tau\right)^{2}}{2}\right] \cdot \exp \left\{-2 a_{1}^{2}\left(\omega_{0} \tau\right)^{2}\right\} \quad\left(\omega_{0} \tau<1\right)
\end{array}
$$

where $\omega_{0}^{2}=\left(\hbar k^{2} / m \varepsilon\right), a_{1}=x_{0} \sqrt{m \omega_{0} / 2 \hbar}$ and $b_{1}=$ $\left(p_{0} / \sqrt{2 m \hbar \omega_{0}}\right)$. As in the nodal case, the damping factor is due to the increasing distance in the phase space of the deflected components of the incoming wavepacket [26].

\section{CONCLUSIONS}

In this paper we have presented a scheme for conditionally teleporting an unknown quantum state between two atoms interacting in succession with the same cavity mode within the optical Stern-Gerlach model. Such model, to be regarded as a generalization of the familiar Jaynes-Cummings Hamiltonian, allows to account for the atomic translational dynamics. The inclusion of such dynamics yields the well-known splitting of the wavefunction of a flying atom into a set of deflected wavepackets. Such phenomenon could be expected to have a negative effect on quantum information processing tasks. Indeed, it is known to spoil the non-local correlations between two atoms which successively interact with the same cavity mode [25, 26]. Nonetheless, in this work we have shown how exactly the above-mentioned splitting can be fruitfully exploited in order for the atomic translational degrees of freedom to behave as channels allowing efficient transmission of quantum information.

Both in the nodal and antinodal case, we have shown that successful teleportation can be obtained with probability $1 / 2$ by measuring the number of cavity photons, the internal state of atom 2 and the position of the two atoms once they are out of the cavity. The teleportation protocol can be therefore implemented through local operations. No direct Bell-state measurements are thus necessary in our scheme. 
The essential requirement for our protocol to work is that the time of flight of each atom inside the cavity is sufficiently long in order which-path information to become accessible. Indeed, the initial wavepacket of each atom splits into a set of outgoing deflected wavepackets which turn out to be orthogonal, and thus distinguishable, provided the atom-cavity interaction time is large enough. Significantly, unlike previous proposals in cavity QED that do not require direct Bell-state measurements, this implies a non holonomous constraint on the atomcavity interaction times. No precise tuning of the atomic flight times inside the cavity is thus needed.

Nonetheless, it should be observed that, in addition, the atom-cavity interaction times must be short enough in order for the lowest-order approximation of the interaction Hamiltonian [Eqs. (3) and (41)] to hold for the whole time of flight of each atom in the cavity. However, this is not a strong constraint. Interaction times of the order of a few Rabi oscillations are indeed enough for a which-path information to be accessed (see the numerical example of Fig. 2 where 6 Rabi oscillations are enough).

To prevent decoherence effects due to the cavity mode damping, it is of course required the total time of the process $t_{3}$ to be shorter than the cavity coherence time $\tau_{c}$. The time $t_{3}$ can be written as $t_{3}=\tau_{1}+\left(t_{2}-t_{1}\right)+\tau_{2}$, where $\tau_{i}$ is the atom-field interaction time for the $i$ th atom and $\left(t_{2}-t_{1}\right)$ represents the time between the exit of atom 1 and the entering of atom 2. Since our protocol does not depend on $\left(t_{2}-t_{1}\right)$ such time can be made as small as allowed by the experimental capabilities. It follows that for all practical purposes it is enough to require that $\tau_{1}+\tau_{2} \ll \tau_{c}$. As pointed out above, each $\tau_{i}$ is required to be larger than a few Rabi oscillations. This also yields a lower bound for $\tau_{c}$ that can be however achieved with present-day technology (see e.g. [8, 34, 35, 36, 37, 38, 39]).

Furthermore, notice that, even though the first and the second atom can be found into, respectively, two and five quantum paths, it is enough to measure only two paths for each atom $\left(l_{i}^{ \pm}\right.$associated with $\left.\left|\Phi_{0, i}^{ \pm}\right\rangle\right)$in order to teleport the initial state of atom 2 into atom 1 . As emphasized in Sec. III, the labeling of such two paths is irrelevant given that it is enough to know only whether the atoms are found in the same path or not. In the latter case, the teleportation can be finalized after a 180 degree rotation around the $z$-axis.

Regarding the position measurements of each atom, these should be performed in such a way not to affect its internal state in the computational space $\{|g\rangle,|e\rangle\}$. This could be accomplished by sending light on the atom of wavelength suitable to excite an atomic transition different from $|g\rangle \leftrightarrow|e\rangle$.

Finally, this work opens the possibility of exploiting the atomic translational degrees of freedom in cavity QED in order to perform other typical quantum information processing tasks, such as the generation of maximally entangled states.

\section{Acknowledgments}

G. Massimo Palma is gratefully acknowledged for fruitful discussions and the critical reading of the manuscript. MT wishes to thank Andreas Buchleitner for fruitful discussions. FC acknowledges support from PRIN 2006 "Quantum noise in mesoscopic systems".

\section{APPENDIX A: DERIVATION OF THE FINAL STATE}

In this Appendix, we describe the procedure for obtaining the state of the system $\left|\Psi\left(t \geq t_{3}\right)\right\rangle$ [Eq. (16)] after that both the atoms have exited the cavity. According to Eq. (8), such state can be obtained through the successive application of operators $U_{\alpha, 1}$ and $U_{\alpha, 2}$ on $|\Psi(0)\rangle[$ Eq. (12)]. We first rewrite the initial state $|\Psi(0)\rangle$ [Eq. (12)] in terms of the dressed states of atom 1 by expressing $\left|e_{1}\right\rangle|0\rangle$ as a linear combination of $\left|\chi_{0,1}^{+}\right\rangle$and $\left|\chi_{0,1}^{-}\right\rangle$. This yields

$$
|\Psi(0)\rangle=\left(\frac{\left|\varphi_{1}(0)\right\rangle\left|\chi_{0,1}^{+}\right\rangle+\left|\varphi_{1}(0)\right\rangle\left|\chi_{0,1}^{-}\right\rangle}{\sqrt{2}}\right)\left|\varphi_{2}(0)\right\rangle\left|\alpha_{2}\right\rangle .
$$

We now let $U_{\alpha, 1}$ act on the initial state (A1) to get $\left|\Psi\left(t_{1}\right)\right\rangle$ (i. e. the state of the system after that atom 1 has exited the cavity). By using (15), we obtain

$$
\begin{aligned}
\left|\Psi\left(t_{1}\right)\right\rangle & =U_{\alpha, 1}|\Psi(0)\rangle= \\
& =\frac{\left|\Phi_{0,1}^{+}\right\rangle\left|\chi_{0,1}^{+}\right\rangle+\left|\Phi_{0,1}^{-}\right\rangle\left|\chi_{0,1}^{-}\right\rangle}{\sqrt{2}}\left|\varphi_{2}(0)\right\rangle\left|\alpha_{2}\right\rangle .
\end{aligned}
$$

Since in the time interval between $t_{1}$ and $t_{2}$, according to Eqs. (5) and (6), $H_{\alpha}^{I}(t)=0(\alpha=N, A)$, it turns out that $\left|\Psi\left(t_{2}\right)\right\rangle=\left|\Psi\left(t_{1}\right)\right\rangle$. Before applying $U_{\alpha, 2}$ to $\left|\Psi\left(t_{2}\right)\right\rangle$ to get $\left|\Psi\left(t_{3}\right)\right\rangle$, it is convenient to rearrange $\left|\Psi\left(t_{2}\right)\right\rangle$ as an expansion in the cavity field Fock states as

$$
\left|\Psi\left(t_{2}\right)\right\rangle=\left|\Psi\left(t_{1}\right)\right\rangle=\left[\frac{\left|\Phi_{0,1}^{+}\right\rangle+\left|\Phi_{0,1}^{-}\right\rangle}{2}\left|e_{1}\right\rangle|0\rangle+\frac{\left|\Phi_{0,1}^{+}\right\rangle-\left|\Phi_{0,1}^{-}\right\rangle}{2}\left|g_{1}\right\rangle|1\rangle\right]\left|\varphi_{2}(0)\right\rangle\left[\cos \frac{\vartheta}{2}\left|e_{2}\right\rangle+e^{i \varphi} \sin \frac{\vartheta}{2}\left|g_{2}\right\rangle\right]
$$

Expanding each state $\left|g_{2}\right\rangle|n\rangle$ and $\left|e_{2}\right\rangle|n\rangle$ in Eq. A3 in terms of $\left|g_{2}\right\rangle|0\rangle$ and of the dressed states atom $2\left|\chi_{0,2}^{ \pm}\right\rangle$ and $\left|\chi_{1,2}^{ \pm}\right\rangle$and, once $U_{\alpha, 2}$ is applied to $\left|\Psi\left(t_{2}\right)\right\rangle$ with the 
help of Eq. (15), the final state of Eq. (16) is obtained.

\section{APPENDIX B: INSIGHT INTO THE MECHANISM BEHIND THE SCHEME}

In the present proposal, quantum information is transferred from atom 2 to atom 1 by using a three-partite continuous-variable (CV) ancillary system that consists of the translational degrees of freedom of both the atoms and the cavity field. Immediately before atom 2 enters the cavity, the state of the system $\left|\Psi\left(t_{2}\right)\right\rangle$, obtained under application of $U_{\alpha, 1}$ onto the initial state (12) [cfr. Eq. (A2) in Appendix A], can be put in the form

$$
\begin{aligned}
\left|\Psi\left(t_{2}\right)\right\rangle= & \frac{\left|\varphi_{2}(0)\right\rangle}{2 \sqrt{2}}\left(\left|\chi_{0,2}^{+}\right\rangle\left|\Phi_{0,1}^{+}\right\rangle\left|\alpha_{1}\right\rangle-\left|\chi_{0,2}^{+}\right\rangle\left|\Phi_{0,1}^{-}\right\rangle \sigma_{z}\left|\alpha_{1}\right\rangle-\left|\chi_{0,2}^{-}\right\rangle\left|\Phi_{0,1}^{+}\right\rangle \sigma_{z}\left|\alpha_{1}\right\rangle+\left|\chi_{0,2}^{-}\right\rangle\left|\Phi_{0,1}^{-}\right\rangle\left|\alpha_{1}\right\rangle\right. \\
& \left.+\left|\xi_{0,2}^{+}\right\rangle\left|\Phi_{0,1}^{+}\right\rangle \sigma_{x}\left|\alpha_{1}\right\rangle-\left|\xi_{0,2}^{-}\right\rangle\left|\Phi_{0,1}^{-}\right\rangle \sigma_{x}\left|\alpha_{1}\right\rangle+\left|\xi_{0,2}^{-}\right\rangle\left|\Phi_{0,1}^{+}\right\rangle i \sigma_{y}\left|\alpha_{1}\right\rangle-\left|\xi_{0,2}^{+}\right\rangle\left|\Phi_{0,1}^{-}\right\rangle i \sigma_{y}\left|\alpha_{1}\right\rangle\right),
\end{aligned}
$$

where $\left|\xi_{0,2}^{ \pm}\right\rangle=\left(\left|e_{2}\right\rangle|1\rangle \pm\left|g_{2}\right\rangle|0\rangle\right) / \sqrt{2}$ are maximally entangled states between the internal degrees of freedom of atom 2 and the cavity field. Notice that the first line of Eq. (B1) contains terms proportional to either $\left|g_{2}\right\rangle|1\rangle$ or $\left|e_{2}\right\rangle|0\rangle$, whereas the states appearing in the second line involve either $\left|g_{2}\right\rangle|0\rangle$ or $\left|e_{2}\right\rangle|1\rangle$. First, the structure of state (B1) shows that, similarly to what has been pointed out in Ref. [18], successful teleportation can, in principle, be achieved with probability 1 . Indeed, provided the interaction time $\tau_{1}$ between the atom 1 and the cavity is large enough that which-path information becomes accessible, $\left|\Phi_{0,1}^{+}\right\rangle$and $\left|\Phi_{0,1}^{-}\right\rangle$become orthogonal [cfr. Sec. III] Eq. (20)]. Therefore the states $\left|\chi_{0,2}^{\eta}\right\rangle\left|\Phi_{0,1}^{\eta^{\prime}}\right\rangle,\left|\xi_{0,2}^{\eta}\right\rangle\left|\Phi_{0,1}^{\eta^{\prime}}\right\rangle$ $\left(\eta, \eta^{\prime}= \pm\right)$, each multiplying $\left|\alpha_{1}\right\rangle$ or $\sigma_{j}\left|\alpha_{1}\right\rangle(j=x, y, z)$, form an orthonormal set. However, direct projections onto maximally entangled states $\left|\chi_{0,2}^{ \pm}\right\rangle$and $\left|\xi_{0,2}^{ \pm}\right\rangle$are expected to be non trivial. In addition, notice that, even assuming the feasibility of a direct measurement of these states, this would not be sufficient to complete the teleportation since states $\left|\Phi_{0,1}^{ \pm}\right\rangle$need to be measured as well. For instance, the first two terms in the right-hand side of Eq. (B1) show that, without measuring the position of atom 1, a measurement outcome $\left|\chi_{0,2}^{+}\right\rangle$does not suffice to conclude whether or not a $\pi$-rotation around the $z$-axis has to be applied to complete the teleportation: if atom 1 is found in path $l_{1}^{-}$this rotation needs to be performed, whereas if atom 1 is found in path $l_{1}^{+}$it is not required. Furthermore, notice that, unlike in the scheme of Ref. [14], each state $\left|\chi_{0,2}^{\eta}\right\rangle\left|\Phi_{0,1}^{\eta^{\prime}}\right\rangle$ and $\left|\xi_{0,2}^{\eta}\right\rangle\left|\Phi_{0,1}^{\eta^{\prime}}\right\rangle$ is not entangled with respect to the internal variables of the atom to be teleported (i.e. atom 1) and the translational degrees of freedom of both atoms (i.e. part of the ancilla). Finally, we point out that, at this stage, translational degrees of freedom of atom 2 do not play any role, yet [notice the common factor $\left|\varphi_{2}(0)\right\rangle$ in Eq. [B1)]

The difficulty of projecting onto entangled states is overcome by applying the second unitary transformation $U_{\alpha, 2}$. Translational degrees of freedom of atom 2 are now involved. Using Eq. (15), the application of $U_{\alpha, 2}$ in fact accomplishes the following mapping

$$
\begin{array}{r}
U_{\alpha, 2}\left|\chi_{0,2}^{\eta}\right\rangle\left|\varphi_{2}(0)\right\rangle\left|\Phi_{0,1}^{\eta^{\prime}}\right\rangle=\left|\chi_{0,2}^{\eta}\right\rangle\left|\Phi_{0,2}^{\eta}\right\rangle\left|\Phi_{0,1}^{\eta^{\prime}}\right\rangle \\
U_{\alpha, 2}\left|\xi_{0,2}^{\eta}\right\rangle\left|\varphi_{2}(0)\right\rangle\left|\Phi_{0,1}^{\eta^{\prime}}\right\rangle=\left[\frac{\eta}{\sqrt{2}}\left|g_{2}\right\rangle|0\rangle\left|\varphi_{2}(0)\right\rangle\right. \\
\left.+\frac{1}{2}\left(\left|\chi_{1,2}^{+}\right\rangle\left|\Phi_{1,2}^{+}\right\rangle+\left|\chi_{1,2}^{-}\right\rangle\left|\Phi_{1,2}^{-}\right\rangle\right)\right]\left|\Phi_{0,1}^{\eta^{\prime}}\right\rangle
\end{array}
$$

We see that the second unitary transformation $U_{\alpha, 2}$ leaves the states $\left|\chi_{0,2}^{ \pm}\right\rangle$unchanged, but, noticeably, attaches a different wavepacket $\left|\Phi_{0,2}^{ \pm}\right\rangle$of atom 2 to each of them. As $\left|\chi_{0,2}^{\eta}\right\rangle=\left(\left|e_{2}\right\rangle|0\rangle \pm|g\rangle|1\rangle\right) / \sqrt{2}$ and looking at the first line of Eq. (B1), it is clear that now distinguishing between $\left|\chi_{0,2}^{-}\right\rangle$and $\left|\chi_{0,2}^{-}\right\rangle$is no longer required to complete the teleportation. In order to assess whether or not a rotation $\sigma_{z}$ has to be applied, it is sufficient to acquire information about the positions of the two atoms. If they are found in paths of equal signs the teleportation is completed already, whereas in the case of paths with opposite signs a further application of $\sigma_{z}$ is needed.

The same phenomenon does not occur for states appearing in the second line of Eq. (B1) due to mapping (B3). Indeed, the application of $U_{\alpha, 2}$ changes both the atom-field maximally entangled states $\left|\xi_{0,2}^{+}\right\rangle$and $\left|\xi_{0,2}^{-}\right\rangle$ yielding terms proportional to either $\left(\sigma_{x}+i \sigma_{y}\right)\left|\alpha_{1}\right\rangle=$ $2 \cos \frac{\vartheta}{2}\left|g_{1}\right\rangle$ or $\left(\sigma_{x}-i \sigma_{y}\right)\left|\alpha_{1}\right\rangle=2 e^{i \varphi} \sin \frac{\vartheta}{2}\left|e_{1}\right\rangle$. No teleportation is therefore achievable in this case.

In summary, as the four terms proportional to states $\left|\chi_{0,2}^{+}\right\rangle$or $\left|\chi_{0,2}^{-}\right\rangle$in Eq. (B1) are those contributing to successful teleportation, their common factor $(2 \sqrt{2})^{-1}$ yields that the probability of success of the scheme is $1 / 2$.

\section{APPENDIX C: PHOTON-NUMBER MEASUREMENTS}

In this Appendix, we present a method to perform the necessary photon-number measurements required by our 
teleportation protocol. This task is achieved through position measurements of a third probe atom $p$.

To complete the protocol, we need to detect the cavityfield Fock states $|0\rangle$ and $|1\rangle$ at $t \geq t_{3}$ (see Sec. III, Table 1 and Fig. 1). According to the initial state (12) and due to the conservation of the free energy $\sum_{i=1,2} S_{z, i}+a^{\dagger} a$, the final state in Eq. (16) at $t \geq t_{3}$ has the form

$$
\left|\psi\left(t_{3}\right)\right\rangle=\sum_{n=0,2}\left|c_{n}\right\rangle_{12}|n\rangle
$$

where $\left|c_{n}\right\rangle_{12}$ are states belonging to the overall Hilbert space of atoms 1 and 2 .

Assume that at $t \geq t_{3}$ the probe atom $p$ is sent through the cavity in the ground state $\left|g_{p}\right\rangle$ and translational wavepacket $\left|\varphi_{p}(0)\right\rangle$, and that it interacts with the field for a time $\tau_{p}$. As $p$ exits the cavity, the final state of the total system has the form

$$
\left|\Psi\left(t_{3}+\tau_{p}\right)\right\rangle=\sum_{n=0,2}\left|c_{n}\right\rangle_{12}\left[U_{\alpha, p}\left|\varphi_{p}(0)\right\rangle\left|g_{p}\right\rangle|n\rangle\right]
$$

where the evolution operator associated with the atom $p$-field dynamics $U_{\alpha, p}$ has a form analogous to Eq. (9) (the integration bounds in this case are obviously $t_{3}$ and $\left.\tau_{p}\right)$. By using Eq. (15), each state $\left|\varphi_{p}(0)\right\rangle\left|g_{p}\right\rangle|n\rangle$ in the right-hand side of Eq. (C2), once expressed in terms of the dressed states $\left|\chi_{n, p}^{ \pm}\right\rangle=\left(\left|e_{p}\right\rangle|n\rangle \pm\left|g_{p}\right\rangle|n+1\rangle\right) / \sqrt{2}$, transforms according to

$$
\begin{gathered}
U_{\alpha, p}\left|\varphi_{p}(0)\right\rangle\left|g_{p}\right\rangle|0\rangle=\left|\varphi_{p}(0)\right\rangle\left|g_{p}\right\rangle|0\rangle, \\
U_{\alpha, p}\left|\varphi_{p}(0)\right\rangle\left|g_{p}\right\rangle|1\rangle=\frac{\left|\Phi_{0}^{+}\right\rangle\left|\chi_{0, p}^{+}\right\rangle-\left|\Phi_{0}^{-}\right\rangle\left|\chi_{0, p}^{-}\right\rangle}{\sqrt{2}}, \\
U_{\alpha, p}\left|\varphi_{p}(0)\right\rangle\left|g_{p}\right\rangle|2\rangle=\frac{\left|\Phi_{1}^{+}\right\rangle\left|\chi_{1, p}^{+}\right\rangle-\left|\Phi_{1}^{-}\right\rangle\left|\chi_{1, p}^{-}\right\rangle}{\sqrt{2}} .
\end{gathered}
$$

Therefore, the unitary operator $U_{\alpha, p}$ in fact maps the Fock states $|0\rangle,|1\rangle$ and $|2\rangle$ into the orthogonal evolved states (C3), (C4) and (C5), respectively. A position measurement of the probe atom, in general, cannot distinguish such states since the wavefunctions $|\varphi(0)\rangle,\left|\Phi_{0}^{ \pm}(x)\right\rangle$ and $\left|\Phi_{1}^{ \pm}(x)\right\rangle$ do not form an orthogonal set. However, provided the interaction time $\varepsilon \tau_{p}$ is large enough, these translational states have a negligible overlap according to

$$
\begin{gathered}
\lim _{\tau_{p} \rightarrow \infty}\left\langle\varphi(0) \mid \Phi_{n}^{\eta}\right\rangle=0 \\
\lim _{\tau_{p} \rightarrow \infty}\left\langle\Phi_{n}^{\eta} \mid \Phi_{n^{\prime}}^{\eta^{\prime}}\right\rangle=0,
\end{gathered}
$$

with $n=0,1,2, \ldots$ and $\eta, \eta^{\prime}= \pm$. Notice that property (20) is a particular case of Eq. (C7).

When the probe atom interacts with the cavity field in a nodal region properties ([C6) and (C7) are explainable as due to the fact that each translational wavepacket $\left|\Phi_{n}^{\eta}\right\rangle$ has an associated acceleration along the $x$-cavity axis $a_{n}^{\eta}$ that depends on both $n$ and $\eta$ according to $a_{n}^{\eta}=-a_{0} \eta \sqrt{n+1}\left[a_{0}=(\hbar k \epsilon / m)\right][23]$. Therefore, provided $\tau_{p}$ is large enough, the wavepackets $\left|\Phi_{n}^{\eta}\right\rangle$ become mutually distinguishable [23].
[1] A. Einstein, B. Podolsky, and N. Rosen, Phys. Rev. 47, 777 (1935).

[2] M. A. Nielsen and I. L. Chuang, Quantum Computation and Quantum Information (Cambridge University Press, Cambridge, U. K., 2000).

[3] C. H. Bennett, G. Brassard, C. Crepeau, R. Jozsa, A. Peres, and W. K. Wootters, Phys. Rev. Lett. 70, 1895 (1993).

[4] D. Bouwmeester, J.-W. Pan, K. Mattle, M. Eibl, H. Weinfurter, and A. Zeilinger, Nature 390, 575 (1997); D. Boschi, S. Branca, F. De Martini, L. Hardy, and S. Popescu, Phys. Rev. Lett. 80, 1121 (1998).

[5] M. A. Nielsen, E. Knill, and R. Laflamme, Nature 396, 52 (1998).

[6] Q. Zhang, et al., Nat. Phys. 2, 678 (2006)

[7] J. F. Sherson, H. Krauter, R. K. Olsson, B. Julsgaard, K. Hammerer, I. Cirac, E. S. Polzik, Nature 443, 557 (2006)

[8] J. M. Raimond, M. Brune, and S. Haroche, Rev. Mod. Phys. 73, 565 (2001).

[9] L. Davidovich, N. Zagury, M. Brune, J. M. Raimond, and S. Haroche, Phys. Rev. A 50, R895 (1994); J. I. Cirac, and A. S. Parkins, Phys. Rev. A 50, R4441 (1994); S. B. Zheng and G. C. Guo, Phys. Lett. A 232, 171 (1997); S. B. Zheng, Opt. Commun. 167, 111 (1999); S. Bose, P. L. Knight, M. B. Plenio, and V. Vedral, Phys. Rev. Lett. 83, 5158 (1999); S. Bandyopadhyay, Phys. Rev. A 62, 012308 (2000).

[10] M. Riebe, et al., Nature 429, 734 (2004); M. D. Barrett, et al., Nature 429, 737 (2004)

[11] L. Vaidman, Phys. Rev. A 49, 1473 (1994)

[12] N.G. de Almeida, R. Napolitano, and M. H. Y. Moussa, Phys. Rev A. 62, 010101(R) (2000)

[13] S.-B. Zheng, Phys. Rev. A 69, 064302 (2004)

[14] L. Ye, and G.-C. Guo, Phys. Rev. A 70, 054303 (2004)

[15] H. J. Carmichael, and B. C. Sanders, Phys. Rev. A, 60, 2497 (1999)

[16] E. Hagley et al., Phys. Rev. Lett. 79, 1 (1997)

[17] W. B. Cardoso, A. T. Avelar, B. Baseia, and N. G. de Almeida Phys. Rev. A 72, 045802 (2005)

[18] R. W. Chhajlany and A. Wojcik, Phys. Rev. A 73, $016302(2006)$

[19] L. Ye, and G.-C. Guo, Phys. Rev. A 73, 016303 (2006)

[20] T. Sleator, T. Pfau, V. Balykin, O. Carnal, and J. 
Mlynek, Phys. Rev. Lett. 68, 1996(1992); C. Tanguy, S. Reynaud, and C. Cohen-Tannoudji, J. Phys. B 17, 4623 (1984); M. Freyberger, and A. M. Herkommer, Phys. Rev. Lett. 72, 1952 1994; A. Vaglica, Phys. Rev. A 54, 3195 (1996)

[21] R. R. Schlicher, Opt. Comm. 70, 97 (1989)

[22] M. Wilkens, Z. Bialynicka-Birula, and P. Meystre, Phys. Rev. A 45, 477 (1992)

[23] A. Vaglica, Phys. Rev. A 58, 3856 (1998); I. Cusumano, A. Vaglica, and G. Vetri, Phys. Rev. A 66, 043408 (2002)

[24] M. Tumminello, A. Vaglica, and G. Vetri, Europhys. Lett. 65, 785 (2004)

[25] M. Tumminello, A. Vaglica, and G. Vetri, Europhys. Lett. 66, 792 (2004)

[26] M. Tumminello, A. Vaglica, and G. Vetri, Eur. Phys. J. D 36, 235 (2005)

[27] A. Vaglica, Phys. Rev. A 52, 2319 (1995)

[28] In the antinodal case, to be pedant, we observe that when the atomic initial wave packet is exactly centered in a field antinode and the initial momentum is 0 then the scalar products in Eq. (20) still decays exponentially but with a slower rate. Accordingly, in this case, several Rabi oscillations are necessary to neglect such scalar products in the protocol [26].

[29] The two atoms can be always distinguished since they exit the cavity at different times.

[30] Similarly to the seminal proposal by Bennett et al. [3], in such cases teleportation is finalized after a 180 degree rotation around the $z$-axis in the internal Hilbert space of atom 1 is performed.

[31] M. Chianello, M. Tumminello, A. Vaglica, and G. Vetri, Phys. Rev. A 69, 053403 (2004)

[32] Y. Aharonov, D.Z. Albert, and C.K. Au, Phys. Rev. Lett. 47, 1029 (1981); R.F. O'Connell and A.K. Rajagopal, Phys. Rev. Lett. 48, 525 (1982)

[33] B.-G. Englert, Phys. Rev. Lett. 77, 2154 (1996)

[34] G. Rempe, et al., Opt. Lett. 7, 363 (1992)

[35] C. J. Hood, H. J. Kimble and J. Ye, Phys. Rev. A 64, 033804 (2001)

[36] H. Mabuchi and A. C. Doherty, Science 298, 1372 (2002)

[37] K. J. Vahala, Nature 424, 839 (2003)

[38] T. Aoki et al., Nature 443, 671 (2006)

[39] S. Kuhr et al., Appl. Phys. Lett. 90, 164101 (2007) 